\title{
BOTTOM SCHUR FUNCTIONS
}

\author{
Peter Clifford \\ CNRI, Dublin Institute of Technology, Ireland \\ petercoalum.mit.edu \\ Richard P. Stanley ${ }^{1}$ \\ Department of Mathematics, Massachusetts Institute of Technology \\ Cambridge, MA 02139, USA \\ rstan@math.mit.edu
}

Submitted: Nov 19, 2003; Accepted: Aug 27, 2004; Published: Sep 24, 2004

MR Subject Classifications: 05E05, 05E10

\begin{abstract}
We give a basis for the space spanned by the sum $\hat{s}_{\lambda}$ of the lowest degree terms in the expansion of the Schur symmetric functions $s_{\lambda}$ in terms of the power sum symmetric functions $p_{\mu}$, where $\operatorname{deg}\left(p_{i}\right)=1$. These lowest degree terms correspond to minimal border strip tableaux of $\lambda$. The dimension of the space spanned by $\hat{s}_{\lambda}$, where $\lambda$ is a partition of $n$, is equal to the number of partitions of $n$ into parts differing by at least 2. Applying the Rogers-Ramanujan identity, the generating function also counts the number of partitions of $n$ into parts $5 k+1$ and $5 k-1$.

We also show that a symmetric function closely related to $\hat{s}_{\lambda}$ has the same coefficients when expanded in terms of power sums or augmented monomial symmetric functions.
\end{abstract}

\section{Introduction}

Let $\lambda=\left(\lambda_{1}, \lambda_{2}, \ldots\right)$ be a partition of the integer $n$, i.e., $\lambda_{1} \geqslant \lambda_{2} \geqslant \cdots \geqslant 0$ and $\sum \lambda_{i}=n$. The length $\ell(\lambda)$ of a partition $\lambda$ is the number of nonzero parts of $\lambda$. The (Durfee or Frobenius) rank of $\lambda$, denoted $\operatorname{rank}(\lambda)$, is the length of the main diagonal of the diagram of $\lambda$, or equivalently, the largest integer $i$ for which $\lambda_{i} \geqslant i$. The rank of $\lambda$ is the least integer $r$ such that $\lambda$ is a disjoint union of $r$ border strips (defined below).

Nazarov and Tarasov [1, Sect. 1], in connection with tensor products of Yangian modules, defined a generalization of rank to skew partitions (or skew diagrams) $\lambda / \mu$. The paper [3, Proposition 2.2] gives several simple equivalent definitions of $\operatorname{rank}(\lambda / \mu)$. One of the definitions is that $\operatorname{rank}(\lambda / \mu)$ is the least integer $r$ such that $\lambda / \mu$ is a disjoint union of $r$ border strips. It develops a general theory of minimal border strip tableaux of skew shapes, introducing the concepts of the snake sequence and the interval set of a skew shape $\lambda / \mu$. These tools are used to count the number of minimal border strip decompositions and minimal border strip tableaux of $\lambda / \mu$. In particular, the paper [3] gives an explicit

\footnotetext{
${ }^{1}$ Partially supported by NSF grant \#DMS-9988459.
} 
combinatorial formula for the coefficients of the $p_{\nu}$, where $\ell(\nu)=\operatorname{rank}(\lambda / \mu)$, which appear in the expansion of $s_{\lambda / \mu}$.

The paper [3] considered a degree operator $\operatorname{deg}\left(p_{\nu}\right)=\ell(\nu)$ and defined the bottom Schur functions to be the sum of the terms of lowest degree which appear in the expansion of $s_{\lambda / \mu}$ as a linear combination of the $p_{\nu}$. We study the bottom Schur functions in detail when $\mu=\emptyset$. In particular, in Section 4 we give a basis for the vector space they span.

In Section 7 we show that when we substitute $i p_{i}$ for $p_{i}$ in the expansion of a bottom Schur function in terms of power sums, then the resulting symmetric function has the same coefficients when expanded in terms of power sums or augmented monomial symmetric functions.

\section{Definitions}

In general we follow $[2, \mathrm{Ch} .7]$ for notation and terminology involving symmetric functions. Let $\lambda$ be a partition of $n$ with Frobenius rank $k$. Recall that $k$ is the length of the main diagonal of the diagram of $\lambda$, or equivalently, the largest integer $i$ for which $\lambda_{i} \geqslant$ $i$. Let $m_{i}(\lambda)=\#\left\{j: \lambda_{j}=i\right\}$, the number of parts of $\lambda$ equal to $i$. Define $z_{\lambda}=$ $1^{m_{1}(\lambda)} m_{1}(\lambda) ! 2^{m_{2}(\lambda)} m_{2}(\lambda) ! \cdots$. A border strip (or rim hook or ribbon) is a connected skew shape with no $2 \times 2$ square. An example is $75443 / 4332$ whose diagram is illustrated in Figure 1. Define the height $\operatorname{ht}(B)$ of a border strip $B$ to be one less than its number of rows.

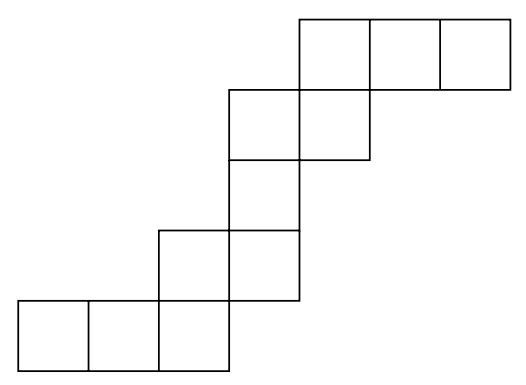

Figure 1: The border strip 75443/4332

Let $\alpha=\left(\alpha_{1}, \alpha_{2}, \ldots\right)$ be a weak composition of $n$, i.e., $\alpha_{i} \geqslant 0$ and $\sum \alpha_{i}=n$. Define a border strip tableau of shape $\lambda$ and type $\alpha$ to be an assignment of positive integers to the squares of $\lambda$ such that:

(a) every row and column is weakly increasing,

(b) the integer $i$ appears $\alpha_{i}$ times, and

(c) the set of squares occupied by $i$ forms a border strip.

Equivalently, one may think of a border-strip tableau as a sequence $\emptyset=\lambda^{0} \subseteq \lambda^{1} \subseteq \cdots \subseteq$ $\lambda^{r} \subseteq \lambda$ of partitions such that each skew shape $\lambda^{i} / \lambda^{i+1}$ is a border-strip of size $\alpha_{i}$. For 


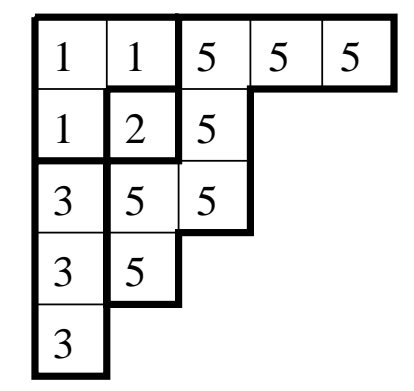

Figure 2: A border strip tableau of 53321 of type $(3,1,3,0,7)$

instance, Figure 2 shows a border strip tableau of 53321 of type $(3,1,3,0,7)$. It is easy to see (in this nonskew case) that the smallest number of strips in a border-strip tableau is $\operatorname{rank}(\lambda)$. Define the height ht $(T)$ of a border-strip tableau $T$ to be

$$
\operatorname{ht}(T)=\operatorname{ht}\left(B_{1}\right)+\operatorname{ht}\left(B_{2}\right)+\cdots+\operatorname{ht}\left(B_{k}\right)
$$

where $B_{1}, \ldots, B_{k}$ are the (nonempty) border strips appearing in $T$. In the example we have $\operatorname{ht}(T)=1+0+2+3=6$. Now we can define

$$
\chi^{\lambda}(\nu)=\sum_{T}(-1)^{\mathrm{ht}(T)},
$$

summed over all border-strip tableaux of shape $\lambda$ and type $\nu$. Since there are at least $\operatorname{rank}(\lambda)$ strips in every tableau, we have that $\chi^{\lambda}(\nu)=0$ if $\ell(\nu)<\operatorname{rank}(\lambda)$. The numbers $\chi^{\lambda}(\nu)$ for $\lambda, \nu \vdash n$ are the values of the irreducible characters $\chi^{\lambda}$ of the symmetric group $S_{n}$.

Finally we can express the Schur function $s_{\lambda}$ in terms of power sums $p_{\nu}$, viz.,

$$
s_{\lambda}=\sum_{\nu} \chi^{\lambda}(\nu) \frac{p_{\nu}}{z_{\nu}}
$$

Define $\operatorname{deg}\left(p_{i}\right)=1$, so $\operatorname{deg}\left(p_{\nu}\right)=\ell(\nu)$. The bottom Schur function $\hat{s}_{\lambda}$ is defined to be the lowest degree part of $s_{\lambda}$, so

$$
\hat{s}_{\lambda}=\sum_{\nu: \ell(\nu)=\operatorname{rank}(\lambda)} \chi^{\lambda}(\nu) \frac{p_{\nu}}{z_{\nu}} .
$$

Also write $\tilde{p}_{i}=\frac{p_{i}}{i}$. For instance,

$$
s_{321}=\frac{1}{45} p_{1}^{6}-\frac{1}{9} p_{3} p_{1}^{3}+\frac{1}{5} p_{1} p_{5}-\frac{1}{9} p_{3}^{2}
$$

Hence

$$
\begin{aligned}
\hat{s}_{321} & =\frac{1}{5} p_{1} p_{5}-\frac{1}{9} p_{3}^{2} \\
& =\tilde{p}_{1} \tilde{p}_{5}-\tilde{p}_{3}^{2} .
\end{aligned}
$$


We identify a partition $\lambda$ with its diagram

$$
\lambda=\left\{(i, j): 1 \leqslant j \leqslant \lambda_{i}\right\} .
$$

Let $e$ be an edge of the lower envelope of $\lambda$, i.e., no square of $\lambda$ has $e$ as its upper or left-hand edge. We will define a certain subset $S_{e}$ of squares of $\lambda$, called a snake. If $e$ is horizontal and $(i, j)$ is the square of $\lambda$ having $e$ as its lower edge, define

$$
\begin{aligned}
S_{e}= & (\lambda) \cap\{(i, j),(i-1, j),(i-1, j-1), \\
& (i-2, j-1),(i-2, j-2), \ldots\} .
\end{aligned}
$$

If $e$ is vertical and $(i, j)$ is the square of $\lambda$ having $e$ as its right-hand edge, define

$$
\begin{aligned}
S_{e}= & (\lambda) \cap\{(i, j),(i, j-1),(i-1, j-1), \\
& (i-1, j-2),(i-2, j-2), \ldots\} .
\end{aligned}
$$

In Figure 3 the nonempty snakes of the shape 533322 are shown with dashed paths through their squares, with a single bullet in the two snakes with just one square. The length $\ell(S)$ of a snake $S$ is one fewer than its number of squares; a snake of length $i-1$ (so with $i$ squares) is call an $i$-snake. Call a snake of even length a left snake if $e$ is horizontal and a right snake if $e$ is vertical. It is clear that the snakes are linearly ordered from lower left to upper right. In this linear ordering, replace a left snake with the symbol $L$, a right snake with $R$, and a snake of odd length with $O$. The resulting sequence (which does not determine $\lambda$ ) is called the snake sequence of $\lambda$, denoted $\operatorname{SS}(\lambda)$. For instance, from Figure 3 we see that

$$
\mathrm{SS}(533322)=\text { LLOOLORROOR. }
$$

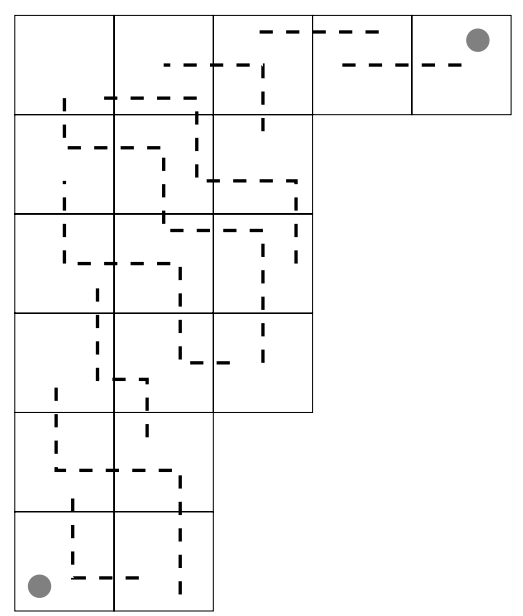

Figure 3: Snakes for the shape 533322

Lemma 2.1. The L's in the snake sequence correspond exactly to horizontal edges of the lower envelope of $\lambda$ which are below the line $x+y=0$. The $R$ 's correspond exactly to vertical edges of the lower envelope of $\lambda$ which are above the line $x+y=0$. All other edges of the lower envelope of $\lambda$ are labelled by $O$ 's. 
Clearly we could have defined the snake sequence this way; however, the definitions above also hold for skew shapes. Lemma 2.1 only holds when $\lambda$ is a straight (i.e., nonskew) shape.

Proof. Let $e$ be an edge of the lower envelope of $\lambda$ below the line $x+y=0$. Let $(i, j)$ be the square of $\lambda$ having $e$ as its lower edge. The last square in the snake is some square in the first column of $\lambda$. So if $e$ is horizontal then the last square is $(i-j+1,1)$, the snake has an odd number of squares and so has even length, and is labelled by $L$. If $e$ is vertical then the last square is $(i-j, 1)$, the snake has an even number of squares, so has odd length, and is labelled by $R$. The case when $e$ is above $x+y=0$ is proved similarly.

Corollary 2.2. In the snake sequence of $\lambda$, the $L$ 's occur strictly to the left of the $R$ 's.

The number of horizontal edges of the lower envelope of $\lambda$ which are below the line $x+y=0$ equals the length of the main diagonal of the diagram of $\lambda$, which is the rank of $\lambda$. Similarly the number of vertical edges of the lower envelope of $\lambda$ which are above the line $x+y=0$ also equals the rank of $\lambda$. Henceforth we fix $k=\operatorname{rank}(\lambda)$.

Let $\operatorname{SS}(\lambda)=q_{1} q_{2} \cdots q_{m}$, and define an interval set of $\lambda$ to be a collection $\mathcal{I}$ of $k$ ordered pairs,

$$
\mathcal{I}=\left\{\left(u_{1}, v_{1}\right), \ldots,\left(u_{k}, v_{k}\right)\right\}
$$

satisfying the following conditions:

(a) the $u_{i}$ 's and $v_{i}$ 's are all distinct integers,

(b) $1 \leqslant u_{i}<v_{i} \leqslant m$,

(c) $q_{u_{i}}=L$ and $q_{v_{i}}=R$.

Figure 4 illustrates the interval set $\{(1,11),(2,7),(5,8)\}$ of the shape 533322 .

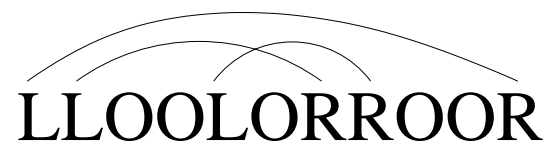

Figure 4: An interval set of the shape 533322

Given an interval set $\mathcal{I}=\left\{\left(u_{1}, v_{1}\right), \ldots,\left(u_{k}, v_{k}\right)\right\}$, define the crossing number $c(\mathcal{I})$ to be the number of crossings of $\mathcal{I}$, i.e. the number of pairs $(i, j)$ for which $u_{i}<u_{j}<v_{i}<v_{j}$.

Let $\boldsymbol{T}$ be a border strip tableau of shape $\lambda$. Recall that

$$
\operatorname{ht}(\boldsymbol{T})=\sum_{B} \mathrm{ht}(B)
$$

where $B$ ranges over all border strips in $\boldsymbol{T}$ and ht $(B)$ is one less than the number of rows of $B$. Define $z(\lambda)$ to be the height ht $(\boldsymbol{T})$ of a "greedy border strip tableau" $\boldsymbol{T}$ of shape $\lambda$ obtained by starting with $\lambda$ and successively removing the largest possible border strip. 
(Although $\boldsymbol{T}$ may not be unique, the set of border strips appearing in $\boldsymbol{T}$ is unique, so $\mathrm{ht}(\boldsymbol{T})$ is well-defined.)

The connection between bottom Schur functions and interval sets was given by Stanley [3, Theorem 5.2]:

$$
\hat{s}_{\nu}=(-1)^{z(\nu)} \sum_{\mathcal{I}=\left\{\left(u_{1}, v_{1}\right), \ldots,\left(u_{k}, v_{k}\right)\right\}}(-1)^{c(\mathcal{I})} \prod_{i=1}^{k} \tilde{p}_{v_{i}-u_{i}},
$$

where $\mathcal{I}$ ranges over all interval sets of $\nu$.

For example the shape 321 has snake sequence LOLROR. There are two interval sets, $\{(1,4),(3,6)\}$ with crossing number 1 , and $\{(1,6),(3,4)\}$ with crossing number 0 . So as we saw before

$$
\hat{s}_{321}=\tilde{p}_{1} \tilde{p}_{5}-\tilde{p}_{3}^{2} .
$$

\section{Bottom Schur Functions of straight shapes}

Lemma 3.1. The lexicographic order on shapes $\nu$ whose length $\ell(\nu)$ equals their rank $k$ is equal to the reverse lexicographical order (with respect to the ordering $L<R<O$ ) on their snake sequences.

Proof. Since $\ell(\nu)=k$, the snake sequence begins with $k$ L's. If the length of the $i$ th row of $\nu$ is $k+j$, then there are $j$ O's to the left of the $(k-i+1)$ st R.

Denote the complete homogeneous symmetric functions by $h_{\lambda}$. Recall that the JacobiTrudi identity expresses the $s_{\lambda}$ 's in terms of the $h_{\mu}$ 's:

$$
s_{\lambda}=\operatorname{det}\left(h_{\lambda_{i}-i+j}\right)_{i, j=1}^{n},
$$

where we define $h_{i}=0$ for $i<0$. For example

$$
s_{554421}=\operatorname{det}\left[\begin{array}{llllll}
h_{5} & h_{6} & h_{7} & h_{8} & h_{9} & h_{10} \\
h_{4} & h_{5} & h_{6} & h_{7} & h_{8} & h_{9} \\
h_{2} & h_{3} & h_{4} & h_{5} & h_{6} & h_{7} \\
h_{1} & h_{2} & h_{3} & h_{4} & h_{5} & h_{6} \\
0 & 0 & 1 & h_{1} & h_{2} & h_{3} \\
0 & 0 & 0 & 0 & 1 & h_{1}
\end{array}\right] .
$$

Since $h_{n}=\sum_{\lambda \vdash n} \frac{p_{\lambda}}{z_{\lambda}}$, the term of lowest degree (in $p$ ) in the expansion of a given $h_{n}$ in terms of the $p_{j}$ is just $\frac{p_{n}}{n}=\tilde{p}_{n}$. For a product $h_{n_{1}} h_{n_{2}} \cdots h_{n_{j}}$ the term of lowest degree in the expansion in terms of the $p_{j}$ is just $\tilde{p}_{n_{1}} \tilde{p}_{n_{2}} \cdots \tilde{p}_{n_{j}}$. So we have that $\hat{s}_{\lambda}=$ terms of lowest order in $\operatorname{det}\left(\tilde{p}_{\lambda_{i}-i+j}\right)_{i, j=1}^{n}$ (since the $p_{\lambda}$ are algebraically independent, and since 
$\operatorname{det}\left(h_{\lambda_{i}-i+j}\right)=s_{\lambda} \neq 0$, this determinant will not vanish). For example

$$
\hat{s}_{554421}=\text { terms of lowest order in } \operatorname{det}\left[\begin{array}{llllll}
\tilde{p}_{5} & \tilde{p}_{6} & \tilde{p}_{7} & \tilde{p}_{8} & \tilde{p}_{9} & \tilde{p}_{10} \\
\tilde{p}_{4} & \tilde{p}_{5} & \tilde{p}_{6} & \tilde{p}_{7} & \tilde{p}_{8} & \tilde{p}_{9} \\
\tilde{p}_{2} & \tilde{p}_{3} & \tilde{p}_{4} & \tilde{p}_{5} & \tilde{p}_{6} & \tilde{p}_{7} \\
\tilde{p}_{1} & \tilde{p}_{2} & \tilde{p}_{3} & \tilde{p}_{4} & \tilde{p}_{5} & \tilde{p}_{6} \\
0 & 0 & 1 & \tilde{p}_{1} & \tilde{p}_{2} & \tilde{p}_{3} \\
0 & 0 & 0 & 0 & 1 & \tilde{p}_{1}
\end{array}\right] \text {. }
$$

Since $p_{0}=1$, the terms of lowest order are those which contain the most number of 1 's.

Row $i$ of the matrix will have a 1 in position $(i, j)$ if $\lambda_{i}-i+j=0$, i.e. if $\lambda_{i}<i$ (this shows that the number of rows of $J T_{\lambda}$ which do not contain a 1 is another definition of $\operatorname{rank}(\lambda)[3$, Prop. 2.2]).

Let $J T_{p}^{*}$ be the matrix obtained from the original Jacobi-Trudi matrix by removing every row and column which contains a 1 and replacing the $h_{i}$ with $\tilde{p}_{i}$. We show below that this matrix is not singular and so we have

$$
\hat{s}_{\lambda}=\operatorname{det} J T_{p}^{*} .
$$

For example

$$
\hat{s}_{554421}=\operatorname{det}\left[\begin{array}{llll}
\tilde{p}_{5} & \tilde{p}_{6} & \tilde{p}_{8} & \tilde{p}_{10} \\
\tilde{p}_{4} & \tilde{p}_{5} & \tilde{p}_{7} & \tilde{p}_{9} \\
\tilde{p}_{2} & \tilde{p}_{3} & \tilde{p}_{5} & \tilde{p}_{7} \\
\tilde{p}_{1} & \tilde{p}_{2} & \tilde{p}_{4} & \tilde{p}_{6}
\end{array}\right] .
$$

Any minor of the Jacobi-Trudi matrix for a shape $\lambda$ is the Jacobi-Trudi matrix for some skew shape $\mu / \sigma$. For let $J T^{*}$ be some minor of size $m$ of some Jacobi-Trudi matrix $J T$. If the entry in position $(i, j)$ is $h_{x}$ put $j t_{i, j}^{*}=x$. Now we can set

$$
\sigma_{i}=j t_{1, m}^{*}-j t_{1, i}^{*}-m+i,
$$

and

$$
\mu_{i}=j t_{i, i}^{*}+\sigma_{i} .
$$

Again note that since the $p_{\lambda}$ are algebraically independent and $\operatorname{det} J T^{*}=s_{\mu / \sigma} \neq 0$, we have det $J T_{p}^{*} \neq 0$.

In our running example, we have $\sigma_{1}=10-5-4+1=2, \sigma_{2}=10-6-4+2=$ $2, \sigma_{3}=10-8-4+3=1$ and $\sigma_{4}=10-10-4+4=0$. Hence $\sigma=(2210)$. Also $\mu_{1}=5+2, \mu_{2}=5+2, \mu_{3}=5+1$ and $\mu_{4}=6+0$. Thus $\mu=(7766)$. Therefore we have that $\hat{s}_{554421}$ equals the determinant of the Jacobi-Trudi matrix of $7766 / 2210$ with the $h$ 's replaced by $\tilde{p}$ 's.

Lemma 3.2. If the skew shape $\mu / \sigma$ has the Jacobi-Trudi matrix $J T^{*}$ obtained by removing all rows and columns with a 1 from a Jacobi-Trudi matrix JT of a shape $\lambda$ with rank $k$, then $\mu / \sigma$ contains a square of size $k$. 
The rank of 554421 is 4 , and the diagram of $7766 / 2210$ does indeed contain a square of size 4:

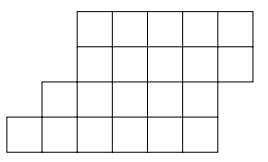

Proof. We give a proof due to Christine Bessenrodt, greatly improving our original proof. Define $\mu_{i}^{\prime}=\ell(\lambda)-k+\lambda_{i}(i=1, \ldots, k)$ and $\sigma_{i}^{\prime}=\#\left\{s \mid \lambda_{s} \leqslant k-i\right\} \quad(i=1, \ldots, k)$. We give a diagrammatic definition of $\mu^{\prime}$ and $\sigma^{\prime}$ which also illustrates that the skew diagram $\mu^{\prime} / \sigma^{\prime}$ contains a square of size $k$. Consider $\lambda$ as a $k \times k$ square with two partitions $\alpha$ and $\beta$ glued to it, i.e. $\lambda=\left(k+\beta_{1}, \ldots, k+\beta_{k}, \alpha_{1}, \ldots, \alpha_{\ell(\lambda)-k}\right)$. Flip $\alpha$ over its anti-diagonal and then glue the bottom right corner of the result to the bottom left corner of the square. The final diagram is the skew diagram of $\mu^{\prime} / \sigma^{\prime}$. We show that $\mu=\mu^{\prime}$ and $\sigma=\sigma^{\prime}$.

The $k$ rows of $J T^{*}$ are contained in the first $k$ rows of $J T$, so $\mu_{i}=\lambda_{i}+c$ for some constant $c$. The last column of JT does not have a 1 in it, so it will not be removed, and its first $k$ entries will be the last column of $J T^{*}$. Hence $j t_{1, k}^{*}=j t_{1, \ell(\lambda)}=\lambda_{1}+\ell(\lambda)-1$. Since $j t_{1, k}^{*}=\mu_{1}-\sigma_{k}+k-1$, we have $\mu_{i}=\ell(\lambda)-k+\lambda_{i}=\mu_{i}^{\prime}$.

The first $k$ entries of the last column of $J T$ are retained. Then we remove the next $\#\left\{s \mid \lambda_{s}=1\right\}$ columns to its left, do not remove the next column, remove the next $\#\left\{s \mid \lambda_{s}=\right.$ $2\}$ columns to the left, and so on. Formally we have $j t_{1, k-j}^{*}=j t_{1, k-j+1}^{*}-1-\#\left\{s \mid \lambda_{s}=j\right\}$. Combining this with $\sigma_{i}=j t_{1, k}^{*}-j t_{1, i}^{*}-k+i$ gives us $\sigma_{i}=\#\left\{s \mid \lambda_{s} \leqslant k-i\right\}=\sigma_{i}^{\prime}$.

\section{The space spanned by the bottom Schur functions}

Before we use the above results to give a basis for the space spanned by the bottom Schur functions, we must first recall some classical tableaux theory.

If $\lambda / \mu$ is a skew shape, then a standard Young tableau (SYT) of shape $\lambda / \mu$ is a labelling of the squares of $\lambda / \mu$ with the numbers $1,2, \ldots, n$, each number appearing once, so that every row and column is increasing. A semistandard Young tableau (SSYT) of shape $\lambda / \mu$ is a labelling of the squares of $\lambda / \mu$ with positive integers that is weakly increasing in every row and strictly increasing in every column. We say that $T$ has type $\alpha=\left(\alpha_{1}, \alpha_{2}, \ldots\right)$ if $T$ has $\alpha_{i}$ parts equal to $i$.

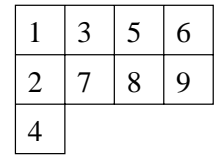

SYT

\begin{tabular}{|l|l|l|l|}
\hline 1 & 1 & 3 & 3 \\
\hline 2 & 4 & 4 & 4 \\
\hline 5 & \multicolumn{4}{|l}{} \\
\cline { 1 - 2 } &
\end{tabular}

SSYT

Now we define an operation (of Schützenberger) on standard Young tableaux called a jeu de taquin slide. Given a skew shape $\lambda / \mu$, consider the squares $b_{0}$ that can be added to 
$\lambda / \mu$, so that $b_{0}$ shares at least one edge with $\lambda / \mu$, and $\left\{b_{0}\right\} \cup \lambda / \mu$ is a valid skew shape. Suppose that $b_{0}$ shares a lower or right edge with $\lambda / \mu$ (the other situation is completely analogous). There is at least one square $b_{1}$ in $\lambda / \mu$ that is adjacent to $b_{0}$; if there are two such squares, then let $b_{1}$ be the one with a smaller entry. Move the entry occupying $b_{1}$ into $b_{0}$. Then repeat this procedure, starting at $b_{1}$. The resulting tableau will be a standard Young tableau. Analogously if $b_{0}$ shares an upper or left edge, the operation is the same except we let $b_{1}$ be the square with the bigger entry from two possibilities. For example we illustrate both situations in Figure 5; the tableau on the right results from playing jeu de taquin beginning at the square marked by a bullet on the tableau on the left (and vice versa).

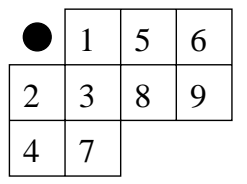

\begin{tabular}{|l|l|l|l|}
\hline 1 & 3 & 5 & 6 \\
\hline 2 & 7 & 8 & 9 \\
\hline 4 & \multicolumn{3}{|l}{} \\
\cline { 1 - 2 } & &
\end{tabular}

Figure 5: Jeu de taquin slides

Two tableaux $T$ and $T^{\prime}$ are called jeu de taquin equivalent if one can be obtained from another by a sequence of jeu de taquin slides. Given an SYT $T$ of shape $\lambda / \mu$, there is exactly one SYT $P$ of straight shape, denoted $\operatorname{jdt}(T)$, that is jeu de taquin equivalent to $T$ [2, Thm. A1.2.4].

The reading word of a (semi)standard Young tableau is the sequence of entries of $T$ obtained by concatenating the rows of $T$ bottom to top. For example, the tableau on the left in Figure 5 has the reading word 472389156. The reverse reading word of a tableau is simply the reading word read backwards.

A lattice permutation is a sequence $a_{1} a_{2} \cdots a_{n}$ such that in any initial factor $a_{1} a_{2} \cdots a_{j}$, the number of $i$ 's is at least as great as the number of $i+1$ 's (for all $i$ ). For example 123112213 is a lattice permutation.

The Littlewood-Richardson coefficients $c_{\mu \nu}^{\lambda}$ are the coefficients in the expansion of a skew Schur function in the basis of Schur functions:

$$
s_{\lambda / \mu}=\sum_{\nu} c_{\mu \nu}^{\lambda} s_{\nu}
$$

The Littlewood-Richardson rule is a combinatorial description of the coefficients $c_{\mu \nu}^{\lambda}$. We will use two different versions of the rule.

Theorem 4.1 (Schützenberger, Thomas). Fix an SYT P of shape $\nu$. The LittlewoodRichardson coefficient $c_{\mu \nu}^{\lambda}$ is equal to the number of $S Y T$ of shape $\lambda / \mu$ that are jeu de taquin equivalent to $P$.

For example, let $\lambda=(5,3,3,1), \mu=(3,1)$, and $\nu=(3,3,2)$. Consider the tableau $P$ of shape $\nu$ shown above. There are exactly two SYTs $T$ of shape $\lambda / \mu$ such that $\operatorname{jdt}(T)=P$, namely, 


$$
P=\begin{array}{|l|l|l|}
\hline 1 & 2 & 3 \\
\hline 4 & 5 & 6 \\
\hline 7 & 8 & \multicolumn{1}{|l}{} \\
\cline { 1 - 2 } &
\end{array}
$$
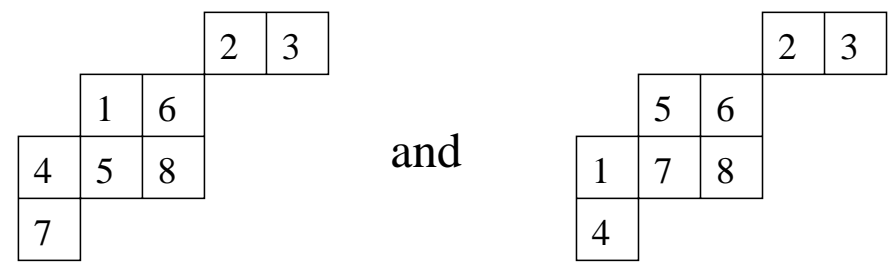

Theorem 4.2 (Schützenberger, Thomas). The Littlewood-Richardson coefficient $c_{\mu \nu}^{\lambda}$ is equal to the number of semistandard Young tableaux of shape $\lambda / \mu$ and type $\nu$ whose reverse reading word is a lattice permutation.

For example, with $\lambda=(5,3,3,1), \mu=(3,1)$, and $\nu=(3,3,2)$ as above, there are exactly two SSYTs $T$ of shape $\lambda / \mu$ and type $\nu$ whose reverse reading word is a lattice permutation:
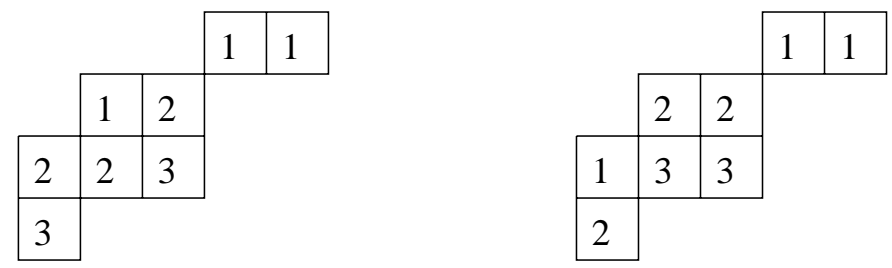

Now we have enough machinery to state and prove this section's main theorem.

Theorem 4.3. Fix $n$ and $k$. The set $\left\{\hat{s}_{\nu}: \nu \vdash n, \operatorname{rank}(\nu)=k\right.$ and $\left.\ell(\nu)=k\right\}$ is a basis for the space $\operatorname{span}_{\mathbb{Q}}\left\{\hat{s}_{\lambda}: \lambda \vdash n\right.$ and $\left.\operatorname{rank}(\lambda)=k\right\}$.

For example if $n=12$ and $k=3$, we have that $\left\{\hat{s}_{633}, \hat{s}_{543}, \hat{s}_{444}\right\}$ is a basis for $\operatorname{span}_{\mathbb{Q}}\left\{\hat{s}_{633}, \hat{s}_{543}, \hat{s}_{5331}, \hat{s}_{444}, \hat{s}_{4431}, \hat{s}_{4332}, \hat{s}_{43311}, \hat{s}_{3333}, \hat{s}_{33321}, \hat{s}_{333111}\right\}$.

Proof. First we prove that the $\hat{s}_{\nu}$ are linearly independent. We show that given any such $\nu$, there is some term in the expansion of $\hat{s}_{\nu}$ which does not occur in the expansion of any $\hat{s}_{\nu^{\prime}}$ for $\nu^{\prime}$ lexicographically less than $\nu$.

From [3, Theorem 5.2] we have that

$$
\hat{s}_{\nu}=(-1)^{z(\nu)} \sum_{\mathcal{I}=\left\{\left(u_{1}, v_{1}\right), \ldots,\left(u_{k}, v_{k}\right)\right\}}(-1)^{c(\mathcal{I})} \prod_{i=1}^{k} \tilde{p}_{v_{i}-u_{i}}
$$

where $\mathcal{I}$ ranges over all interval sets of $\nu$. Let $t= \pm p_{j_{1} \geqslant \cdots \geqslant j_{k}}$ be the term corresponding to the noncrossing interval set $\mathcal{I}$ of the snake sequence of $\nu$. We claim that $t$ does not occur in the expansion of any $\hat{s}_{\nu^{\prime}}$ for $\nu^{\prime}$ lexicographically less than $\nu$. Assume by way of contradiction that it does occur for some such $\nu^{\prime}$ with corresponding interval set $\mathcal{I}^{\prime}$. 
Assume inductively that the first $i-1$ L's are matched with the last $i-1$ R's without crossings in $\mathcal{I}^{\prime}$. Let $r_{j}$ (and $r_{j}^{\prime}$ respectively) be the position of the $j$ th $\mathrm{R}$ in the snake sequence of $\nu$ ( $\nu^{\prime}$ respectively). By Lemma 3.1 $r_{j} \geqslant r_{j}^{\prime}$. But the length of the interval matching the $i$ th $\mathrm{R}$ from the right in $\mathcal{I}$ is $r_{k-i+1}-i$. So for there to be an interval of this length in $\mathcal{I}^{\prime}$ we must match the $i$ th $\mathrm{R}$ from the right with the $i$ th $\mathrm{L}$; this interval has no crossing. Proceeding by induction we see that $\mathcal{I}^{\prime}$ is also noncrossing, and so must equal $\mathcal{I}$. This shows that the snake sequences corresponding to $\nu$ and $\nu^{\prime}$ are equal. Identical snake sequences and equal lengths guarantee that $\nu=\nu^{\prime}$, a contradiction.

Now we prove that the $\hat{s}_{\nu}$ span the space of all $\hat{s}_{\lambda}$. We have shown that $\hat{s}_{\lambda}=\hat{s}_{\mu / \sigma}$. Expand $s_{\mu / \sigma}$ in terms of (straight) Schur functions using the Littlewood Richardson rule

$$
s_{\mu / \sigma}=\sum_{\nu} c_{\sigma \nu}^{\mu} s_{\nu}
$$

We need to show that $c_{\sigma \nu}^{\mu}=0$ unless $\nu$ is of rank $k$ and length $k$.

Fix an SYT P of shape $\nu$. The Littlewood-Richardson coefficient $c_{\sigma \nu}^{\mu}$ is equal to the number of SYT of shape $\mu / \sigma$ that are jeu de taquin equivalent to P. Playing jeu de taquin on a straight-shape tableau of shape $\nu$ can only increase the length of the shape. Hence if $c_{\sigma \nu}^{\mu} \neq 0, \ell(\nu) \leqslant \ell(\mu)=k$.

The Littlewood-Richardson coefficient $c_{\sigma \nu}^{\mu}$ is also equal to the number of semistandard Young tableaux of shape $\mu / \sigma$ and type $\nu$ whose reverse reading word is a lattice permutation. But we know that $\mu / \sigma$ contains a square of size $k$ (by Lemma 3.2). Therefore the bottom $k$ boxes of this square must have labels at least $k$. Since $\ell(\nu) \leqslant k$, the labels are exactly $k$. So $\nu_{k} \geqslant k$, i.e. $\operatorname{rank}(\nu) \geqslant k$. Since $\ell(\nu) \leqslant k$, we must have $\operatorname{rank}(\nu)=\ell(\nu)=k$.

Taking terms of lowest degree on both sides of

$$
s_{\mu / \sigma}=\sum_{\nu} c_{\sigma \nu}^{\mu} s_{\nu}
$$

we have that

$$
\hat{s}_{\lambda}=\hat{s}_{\mu / \sigma}=\sum_{\nu} c_{\sigma \nu}^{\mu} \hat{s}_{\nu}
$$

where the sum is over $\nu$ of length $k$ and rank $k$ as required.

\section{Dimension of the space spanned by the bottom Schur functions}

Let $p_{\leqslant k}(n)$ be the number of partitions of $n$ with length at most $k$, and define $p_{\leqslant k}(0)=1$. A partition $\nu \vdash n$ of length $k$ and rank $k$ decomposes into a $k \times k$ square of boxes and a partition of $n-k^{2}$ of length at most $k$.

Corollary 5.1. The dimension of the space of bottom Schur functions $\operatorname{span}_{\mathbb{Q}}\left\{\hat{s}_{\lambda}: \lambda \vdash n\right\}$ is

$$
\sum_{k=1}^{\lfloor\sqrt{n}\rfloor} p_{\leqslant k}\left(n-k^{2}\right) .
$$


For example, the first 27 terms in this sequence (beginning with $n=1$ ) are

$$
1,1,1,2,2,3,3,4,5,6,7,9,10,12,14,17,19,23,26,31,35,41,46,54,61,70,79 .
$$

There is a nice bijection between the above partitions and the set of partitions $\{\lambda \vdash n$ : $\left.\lambda_{i}-\lambda_{i+1} \geqslant 2\right\}$. For, given a $k$ and a partition $\lambda^{*} \vdash n-k^{2}$ with fewer than $k$ parts, we can set $\lambda_{i}=\lambda_{i}^{*}+2 k-2 i+1$. This gives a partition of $n$ with $k$ rows with $\lambda_{i}-\lambda_{i+1} \geqslant 2$ as required. This is clearly a bijection.

This classical sequence also gives the number of partitions of $n$ into parts congruent to 1 or $4 \bmod 5$; equivalently these numbers are the coefficients in the expansion of the Rogers-Ramanujan identity

$$
1+\sum_{n \geqslant 1} \frac{t^{n^{2}}}{(1-t)\left(1-t^{2}\right) \cdots\left(1-t^{n}\right)}=\prod_{n \geqslant 1} \frac{1}{\left(1-t^{5 n-1}\right)\left(1-t^{5 n-4}\right)}
$$

\section{2-bottom Schur functions}

We have shown that a basis for the space spanned by the bottom Schur functions consists of the $\hat{s}_{\lambda}$ where $\ell(\lambda) \leqslant \operatorname{rank}(\lambda)$. It is natural to define for fixed $j \geqslant 1$ the $j$-bottom Schur function $\hat{s}_{\lambda}^{j}$ to be the sum of those terms of degree at most $\operatorname{rank}(\lambda)+j-1$ in the expansion (2.1) (with $\operatorname{deg} p_{i}=1$ as usual). When $j=2$ we have verified (using Stembridge's SF package for Maple [4]) that for $n \leqslant 14$ the dimension of the space spanned by $\left\{\hat{s}_{\lambda}^{2}: \lambda \vdash n\right\}$ equals the number of $\lambda \vdash n$ satisfying $\ell(\lambda) \leqslant \operatorname{rank}(\lambda)+1$. This suggests the following conjecture.

Conjecture 6.1. A basis for the space spanned by the 2-bottom Schur functions consists of all 2-bottom Schur functions $\hat{s}_{\lambda}^{2}$, where $\lambda$ is a partition of $n$ satisfying $\ell(\lambda) \leqslant \operatorname{rank}(\lambda)+1$.

However in the $j=3$ case, the dimensions of the spaces spanned by the 3 -bottom Schur functions are $1,2,3,4,6,9,11,15,19,24,30, \ldots$. We have computed that the numbers of $\lambda \vdash n$ satisfying $\ell(\lambda) \leqslant \operatorname{rank}(\lambda)+2$ are given by $1,2,3,4,5,8,10,14,17,22,27, \ldots$ Unfortunately these sequences do not agree.

\section{$7 \quad$ A condition satisfied by bottom Schur functions}

We prove a surprising identity satisfied by a variant of the bottom Schur functions related to their expansion in terms of power sum and monomial symmetric functions.

Fix a shape $\lambda$ of rank $k$. Given an interval set $\mathcal{I}=\left\{\left(u_{1}, v_{1}\right), \ldots,\left(u_{k}, v_{k}\right)\right\}$, of $\lambda$ and a labelling of the intervals $\left(\alpha_{1}, \ldots, \alpha_{k}\right)$ such that $\alpha_{i} \in \mathbb{P}$, define

$$
x^{\mathcal{I}}=\prod_{i=1}^{k} x_{\alpha_{i}}^{v_{i}-u_{i}} .
$$

Recall that $c(\mathcal{I})$ is the number of crossings of the interval set $\mathcal{I}$. Figure 6 shows a labelled interval set of the shape 533322 with the snake sequence LLOOLORROOR. For this interval set $c(\mathcal{I})=1$ and for this labelling $x^{\mathcal{I}}=x_{4}^{10} x_{2}^{5} x_{4}^{3}=x_{2}^{5} x_{4}^{13}$. 


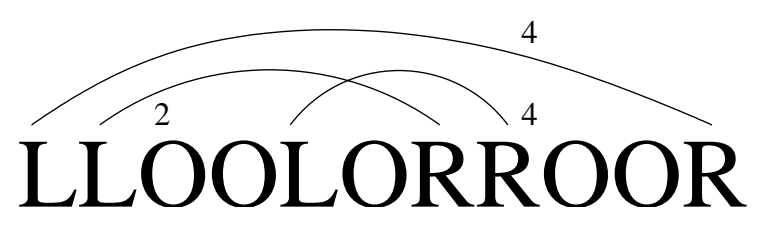

Figure 6: A labelled interval set of the shape 533322

Example 7.1. For the shape $\lambda=(4,4,4)$ with snake sequence $L L L O R R R$, Figure 7 depicts some of the labelled interval sets. In the top left we have $(-1)^{c(\mathcal{I})} x^{\mathcal{I}}=(-1)^{3} x_{a}^{4} x_{a}^{4} x_{b}^{4}=$ $-x_{a}^{8} x_{b}^{4}$. In the top right we have $(-1)^{c(\mathcal{I})} x^{\mathcal{I}}=(-1)^{2} x_{a}^{5} x_{a}^{3} x_{b}^{4}=x_{a}^{8} x_{b}^{4}$. In fact in every row the term $(-1)^{c(\mathcal{I})} x^{\mathcal{I}}$ in the left column is exactly the negative of the corresponding term $(-1)^{c(\mathcal{I})} x^{\mathcal{I}}$ in the right column.

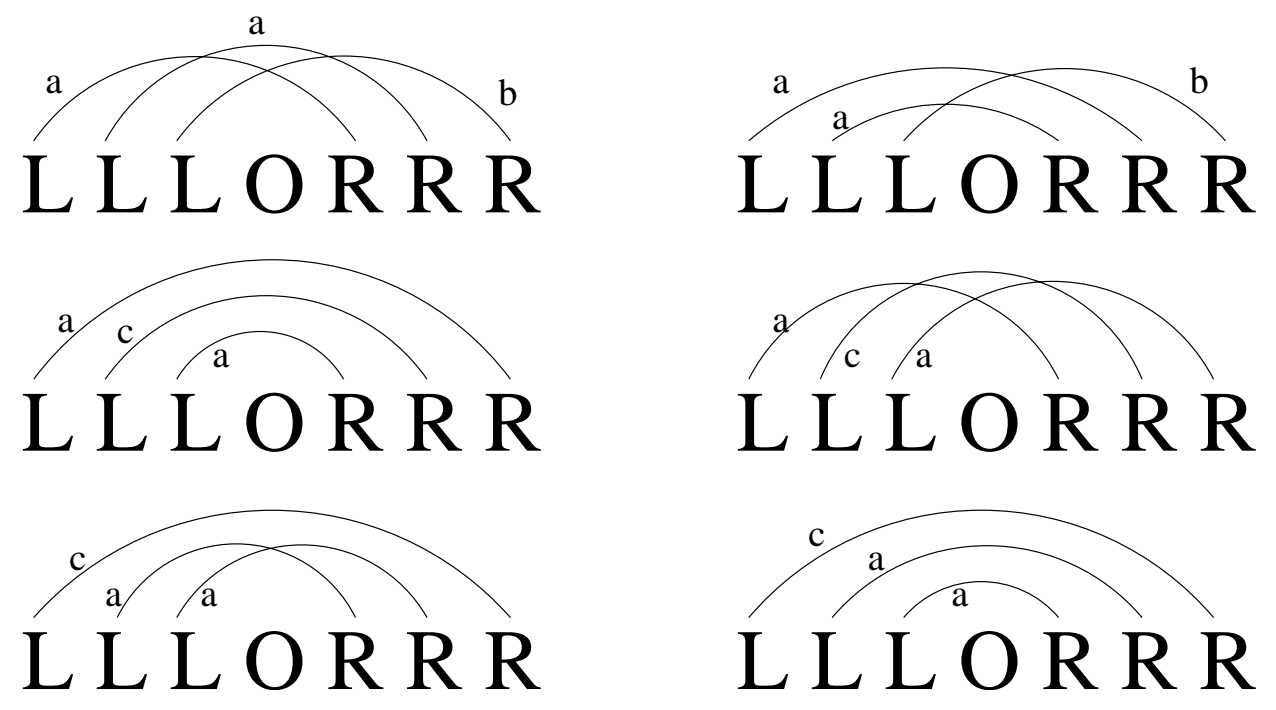

Figure 7: Some labelled interval sets of the shape 444

Lemma 7.1. Fix a shape $\lambda$. Then $\sum(-1)^{c(\mathcal{I})} x^{\mathcal{I}}=0$, where the sum is over all labelled interval sets of $\lambda$ with at least one label repeated.

Proof. We give a sign reversing involution on these labelled interval sets. Examine a specific labelled interval set $\mathcal{I}$. Since we are dealing with straight (non-skew) shapes, we know by Corollary 2.2 that the snake sequence has all the L's before any of the R's, or $u_{k}<v_{1}$. So any two intervals $i$ and $j$ ( $>i$ say) either intersect $\left(u_{i}<u_{j}<v_{i}<v_{j}\right)$ or are nested $\left(u_{i}<u_{j}<v_{j}<v_{i}\right)$.

Let $a$ be the smallest label which is repeated. The intervals in $\mathcal{I}$ are ordered by where they start, so identifying the first two intervals $i$ and $j(>i)$ labelled by $a$ is well-defined.

Simply change the interval $\left(u_{i}, v_{i}\right)$ to $\left(u_{i}, v_{j}\right)$ and the interval $\left(u_{j}, v_{j}\right)$ to $\left(u_{j}, v_{i}\right)$, while preserving the label $a$ on both. Where the intervals start remains unchanged, so these intervals remain the first two intervals labelled by $a$. Hence this operation is an involution. 
Note that if the two intervals initially nested, they now intersect, and if they initially intersected, they now nest. The parity of the number of crossings of these two with any other interval is preserved under this operation. So the parity of the total number of crossings has changed, and this involution is sign reversing. The rows of Figure 7 are some examples of this involution (if $a \neq c$ ).

Thus given any labelled interval set with repeated labels, there is a unique labelled interval set with one more (or fewer) crossings, and so the sum of all such terms $(-1)^{c(\mathcal{I})} x^{\mathcal{I}}$ is zero.

Fix a shape $\lambda$. Recall that

$$
\hat{s}_{\lambda}=(-1)^{z(\lambda)} \sum_{\mathcal{I}=\left\{\left(u_{1}, v_{1}\right), \ldots,\left(u_{k}, v_{k}\right)\right\}}(-1)^{c(\mathcal{I})} \prod_{i=1}^{k} \tilde{p}_{v_{i}-u_{i}}
$$

where $\mathcal{I}$ ranges over all interval sets of $\lambda$.

For another shape $\mu$, define $c_{\mu}$ to be the coefficient of $\tilde{p}_{\mu}$ in the above sum, i.e.

$$
c_{\mu}=(-1)^{z(\lambda)} \sum_{\mathcal{I}}(-1)^{c(\mathcal{I})},
$$

where the sum is over all interval sets (of $\lambda$ ) of type $\mu$.

Lemma 7.2. $c_{\mu} p_{\mu}=(-1)^{z(\lambda)} \sum(-1)^{c(\mathcal{I})} x^{\mathcal{I}}$, where the sum is over all labelled interval sets of type $\mu$.

Example 7.2. Consider as before the shape $\lambda=(4,4,4)$ with snake sequence $L L L O R R R$. In particular, consider the interval set $\{(1,7),(2,5),(3,6)\}$ of type $(6,3,3)$ labelled by $(a, b, c)$. This interval set is illustrated in Figure 8. If $a=b=c$ then $x^{\mathcal{I}}=x_{a}^{12}$ and the sum over all such labellings will give $x_{1}^{12}+x_{2}^{12}+\cdots=m_{(12)}$. If $a=b \neq c$ the sum over all such labellings will give $x_{1}^{6} x_{1}^{3} x_{2}^{3}+x_{1}^{6} x_{1}^{3} x_{3}^{3}+x_{2}^{6} x_{2}^{3} x_{1}^{3}+\cdots=x_{1}^{9} x_{2}^{3}+x_{1}^{9} x_{3}^{3}+$ $x_{2}^{9} x_{1}^{3}+\cdots=m_{93}$. Similarly if $a=c \neq b$ we will get $m_{93}$, and if $b=c \neq a$ we will get $x_{1}^{6} x_{2}^{3} x_{2}^{3}+x_{2}^{6} x_{1}^{3} x_{1}^{3}+\cdots=2 x_{1}^{6} x_{2}^{6}+\cdots=2 m_{66}$. Finally if the three labels are all different, the sum will give $x_{1}^{6} x_{2}^{3} x_{3}^{3}+x_{1}^{6} x_{3}^{3} x_{2}^{3}+\cdots=2 m_{633}$. So the sum over all such labellings is $m_{(12)}+2 m_{66}+2 m_{93}+2 m_{633}=p_{633}$.

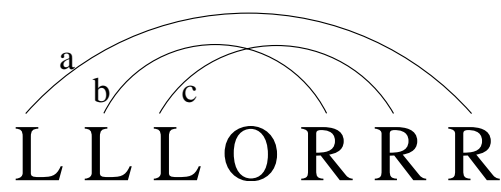

Figure 8: A labelled interval set of the shape 444

Proof. We need to show that for every interval set $\mathcal{I}$ of type $\mu, \sum x^{\mathcal{I}}=p_{\mu}$, where the sum is over all labellings of $\mathcal{I}$. First note that the intervals can be ordered largest first 
and left to right among intervals of the same length. So the $i$ th interval is well defined, and has length $\mu_{i}$.

By definition $p_{\mu}=p_{\mu_{1}} p_{\mu_{2}} \cdots p_{\mu_{\ell(\mu)}}=\left(x_{1}^{\mu_{1}}+x_{2}^{\mu_{1}}+\cdots\right)\left(x_{1}^{\mu_{2}}+x_{2}^{\mu_{2}}+\cdots\right) \cdots\left(x_{1}^{\mu_{\ell(\mu)}}+\right.$ $\left.x_{2}^{\mu_{\ell(\mu)}}+\cdots\right)$. But if we expand this product into monomials $x_{i_{1}}^{\mu_{1}} x_{i_{2}}^{\mu_{2}} \cdots x_{i_{\ell(\mu)}}^{\mu_{\ell(\mu)}}$, each monomial corresponds uniquely to the labelling of $\mathcal{I}$ where the $j$ th interval is labelled by $i_{j}$, and so occurs exactly once in $\sum x^{\mathcal{I}}$ as required.

The augmented monomial symmetric function $\tilde{m}_{\mu}$ is defined by

$$
\tilde{m}_{\mu}=m_{1}(\mu) ! m_{2}(\mu) ! \cdot m_{\mu},
$$

where $m_{\mu}$ denotes a monomial symmetric function.

Lemma 7.3. $c_{\mu} \tilde{m}_{\mu}=(-1)^{z(\lambda)} \sum(-1)^{c(\mathcal{I})} x^{\mathcal{I}}$, where the sum is over all labelled interval sets of $\lambda$ of type $\mu$ with no label repeated.

Note that we have already demonstrated this result in Example 7.2. Indeed for that interval set when the labels were all different we saw that $\sum x^{\mathcal{I}}=2 m_{633}$.

Proof. Fix a specific interval set $\mathcal{I}$ of type $\mu$. We need to show $\tilde{m}_{\mu}=\sum x^{\mathcal{I}}$ where the sum is over all labellings with no label repeated. As before we can order the intervals and say that the $i$ th interval is labelled by $\alpha_{i}$. Note that if $\mu_{j}=\mu_{j+1}$, the two labellings $\left(\alpha_{1}, \alpha_{2}, \ldots, \alpha_{j}, \alpha_{j+1}, \ldots\right)$ and $\left(\alpha_{1}, \alpha_{2}, \ldots, \alpha_{j+1}, \alpha_{j}, \ldots\right)$ both produce the same term $x^{\mathcal{I}}=$ $x_{\alpha_{1}}^{\mu_{1}} x_{\alpha_{2}}^{\mu_{2}} \ldots$. So we have $\sum_{\left(\alpha_{1}, \alpha_{2}, \ldots\right)} x^{\mathcal{I}}=\sum_{\left(\beta_{1}, \beta_{2}, \ldots\right)} m_{1}(\mu) ! m_{2}(\mu) ! \cdots x^{\mathcal{I}}$ where we impose the condition that if $\mu_{j}=\mu_{j+1}$, then $\beta_{j}<\beta_{j+1}$. Recall that $m_{\mu}=\sum_{\left(\beta_{1}, \beta_{2}, \ldots\right)} x^{\mathcal{I}}$ by definition. So we have $\tilde{m}_{\mu}=\sum x_{\left(\alpha_{1}, \alpha_{2}, \ldots\right)}^{\mathcal{I}}$ as required.

Theorem 7.4. For each shape $\lambda$, write the bottom Schur function $\hat{s}_{\lambda}=\sum_{\mu} c_{\mu} \tilde{p}_{\mu}$. Then $\sum_{\mu} c_{\mu} p_{\mu}=\sum_{\mu} c_{\mu} \tilde{m}_{\mu}$.

Example 7.3. For $\lambda=(4,4,4)$ we have

$$
\hat{s}_{\lambda}=-\tilde{p}_{642}+\tilde{p}_{633}+\tilde{p}_{552}-2 \tilde{p}_{543}+\tilde{p}_{444} .
$$

So our result states that

$$
-p_{642}+p_{633}+p_{552}-2 p_{543}+p_{444}=-m_{642}+2 m_{633}+2 m_{552}-2 m_{543}+6 m_{444} .
$$

Proof. From Lemma 7.2 we have $c_{\mu} p_{\mu}=(-1)^{z(\lambda)} \sum(-1)^{c(\mathcal{I})} x^{\mathcal{I}}$, where the sum is over all labelled interval sets of type $\mu$. But by Lemma 7.1

$$
(-1)^{z(\lambda)} \sum(-1)^{c(\mathcal{I})} x^{\mathcal{I}}=0
$$

if we sum over all labelled interval sets with a repeated label, while by Lemma 7.3 we have

$$
(-1)^{z(\lambda)} \sum(-1)^{c(\mathcal{I})} x^{\mathcal{I}}=c_{\mu} m_{1}(\mu) ! m_{2}(\mu) ! \cdots m_{\mu}
$$

if we sum over all labelled interval sets with no label repeated. So $\sum_{\mu} c_{\mu} p_{\mu}=\sum_{\mu} c_{\mu} \tilde{m}_{\mu}$. 
Let $\Gamma$ denote the space of all symmetric functions $f$ with rational coefficients such that if $f=\sum_{\mu} c_{\mu} \tilde{p}_{\mu}$, then

$$
\sum_{\mu} c_{\mu} p_{\mu}=\sum_{\mu} c_{\mu} \tilde{m}_{\mu}
$$

Theorem 7.4 shows that $\hat{s}_{\lambda} \in \Gamma$. However, there are elements of $\Gamma$ that are not linear combinations of $\hat{s}_{\lambda}$ 's, such as $f=\tilde{p}_{511}-3 \tilde{p}_{421}+\tilde{p}_{331}+\tilde{p}_{322}$. Let $R$ denote the transition matrix from monomial symmetric functions to the power sums, i.e.,

$$
p_{\lambda}=\sum_{\mu} R_{\lambda \mu} m_{\mu}
$$

Let $D$ denote the diagonal matrix whose diagonal coincides with that of $R$, so

$$
D_{\lambda \lambda}=R_{\lambda \lambda}=\prod_{i \geqslant 1} m_{i}(\lambda) !
$$

It is easy to see that $\Gamma=\operatorname{ker}\left(R-D\right.$ ), the kernel (or null space) of $R-D$. Let $\Gamma_{n}$ denote the elements of $\Gamma$ that are homogeneous of degree $n$, and let $\gamma_{n}=\operatorname{dim} \Gamma_{n}$. We have computed that

$$
\left(\gamma_{1}, \gamma_{2}, \ldots\right)=(1,1,1,2,2,3,4,5,7,9,11,15,19,24, \ldots) .
$$

Compare this with the dimension $\beta_{n}$ of the space spanned by the bottom Schur functions of degree $n$, given in general by Corollary 5.1 and for $n \leqslant 27$ just below this corollary. In particular, the least $n$ for which $\beta_{n}<\gamma_{n}$ is $n=7$. We don't have a conjecture for the value of $\gamma_{n}$.

\section{References}

[1] M. Nazarov and V. Tarasov, On irreducibility of tensor products of Yangian modules, Internat. Math. Research Notices (1998), 125-150.

[2] R. P. Stanley, "Enumerative Combinatorics," Vol. 2, Cambridge Univ. Press, New York/Cambridge, UK, 1999.

[3] R. P. Stanley, The Rank and Minimal Border Strip Decompositions of a Skew Partition, J. Combin. Theory (A) 100 (2002), 349-375.

[4] J. R. Stembridge, The SF Package for Maple, Version 2.3, 22 July 2001, http://www.math.lsa.umich.edu/ jrs/maple.html\#SF 